\title{
Detection of the Electron Spin Resonance of Two-Dimensional Electrons at Large Wave Vectors
}

\author{
I. V. Kukushkin, ${ }^{1,2}$ J. H. Smet, ${ }^{1}$ D. S. Lyne Abergel, ${ }^{3}$ V. I. Fal'ko, ${ }^{3}$ W. Wegscheider, ${ }^{4,5}$ and K. von Klitzing ${ }^{1}$ \\ ${ }^{1}$ Max-Planck-Institut für Festkörperforschung, Heisenbergstraße 1, 70569 Stuttgart, Germany \\ ${ }^{2}$ Institute of Solid State Physics, RAS, Chernogolovka, 142432 Russia \\ ${ }^{3}$ Physics Department, Lancaster University, LA1 4YB, Lancaster, United Kingdom \\ ${ }^{4}$ Walter Schottky Institut, Technische Universität München, 85748 Garching, Germany \\ ${ }^{5}$ Institut für Experimentelle und Angewandte Physik, Universität Regensburg, 93040 Regensburg, Germany
}

(Received 17 November 2005; published 31 March 2006)

\begin{abstract}
We have investigated the electron spin resonance at nonzero wave vector in GaAs single quantum wells by combining the virtues of high frequency surface acoustic wave generation to produce excitations with large wave numbers with a sensitive optical scheme to detect resonant absorption. The observed large deviations from the single particle Zeeman energy are attributed to the exchange interaction. The enhancement of the electronic $g^{*}$ factor is, however, substantially smaller compared with theoretical predictions for spin waves when adopting a bare Coulomb interaction potential.
\end{abstract}

PACS numbers: 73.20.Mf, 71.36.+c, 73.21.-b, 78.70.Gq

The electron spin resonance (ESR) in a GaAs based twodimensional electron system (2DES) subjected to a perpendicular magnetic field $B$ was first measured in microwave absorption experiments in Refs. [1,2]. The authors resorted to a resistance measurement rather than conventional ESR to detect resonant microwave absorption, as the latter rapidly approaches its sensitivity limitations when the active volume is confined to a nanometer sized region. These experiments obtain a $g^{*}$ factor close to that of bulk GaAs (-0.44), no matter the degree of spin polarization of the electronic system. In contrast, thermally activated transport studies of the 2DES condensed in a quantum Hall state yield a strong filling factor dependent enhancement of $g^{*}$. The enhancement was accounted for by exchange interaction [3] and can be as large as a factor 10-20 at filling $\nu=1$ when all electron spins are aligned [4,5].

The disparity between the outcome of the microwave absorption and transport experiments can readily be reconciled by considering the wave number at which the two experiments probe the spin-flip excitations. The microwaves couple to the center of mass motion and absorption is not accompanied by momentum transfer. Hence, it probes the properties of spin-flip excitations in the limit $k \rightarrow 0$ where their energy is immune to Coulomb interaction phenomena and simply equals the bare Zeeman energy. However, for transport to take place in thermal activation studies, charge separation between the electron promoted to the opposite spin branch of the same Landau level (LL) and the hole it leaves behind is required. Since in a $B$-field spatial separation $r$ is closely related to wave number $k\left(k=r / \lambda_{B}^{2}\right.$, with $\lambda_{B}$ the magnetic length), such investigations address the $k \rightarrow \infty$ limit of the spin-exciton dispersion. Theory predicts that this dispersion starts from the bare Zeeman energy at $k=0$, grows with a $k^{2}$ term and saturates to the sum of the Zeeman energy and the exchange energy as $k$ approaches infinity [6-8]. The exchange interaction strongly oscillates with $\nu$ as its mag- nitude depends on the net electron spin polarization and, of course, scales overall with the inverse of the average interparticle distance $\sqrt{n_{s}}$, where $n_{s}$ is the electron density. Despite general consensus on this theoretical explanation, no experimental attempts have been undertaken to measure the dispersion of spin-excitations away from the two extremes $k \rightarrow 0$ and $k \rightarrow \infty$ in view of the dearth of appropriate techniques. The challenge consists in producing suitable external excitations with wave numbers in the regime close to $2 \pi / \lambda_{B}$ (typically on the order of $10^{8} \mathrm{~m}^{-1}$ ) to probe the 2DES at these wave numbers. Here, we fill this gap by combining high frequency surface acoustic wave (SAW) generation to provide an external excitation at large wave vectors $[9,10]$ with a sensitive optical scheme to detect resonant absorption of the SAWs. With this method, we investigated ESR for $0<$ $\nu<3$ and densities between $3 \times 10^{9} \mathrm{~cm}^{-2}$ and $1.6 \times$ $10^{11} \mathrm{~cm}^{-2}$ at wave numbers up to $4 \times 10^{7} \mathrm{~m}^{-1}$. The exchange contribution at a given wave number is found to be substantially smaller than predicted by theory.

These studies were performed on modulation doped GaAs $/ \mathrm{Al}_{x} \mathrm{Ga}_{1-x}$ As single quantum wells with a well width of $30 \mathrm{~nm}$ and a spacer thickness of $100 \mathrm{~nm}$. The electron density and mobility were measured from the position and the linewidth of dimensional magnetoplasma resonances, which are readily detected in luminescence experiments [11]. The electron density was tuned in the range of $n_{s}=$ $0.3-16 \times 10^{10} \mathrm{~cm}^{-2}$ by illuminating the sample with a HeNe laser (opto-depletion effect). The mobility varied from $0.5 \times 10^{6}$ to $5 \times 10^{6} \mathrm{~cm}^{2} / \mathrm{Vs}$. Mesas with a shape shown in the inset to Fig. 1 were fabricated with optical lithography. Each mesa was composed of a narrow active region, $1 \mathrm{~mm}$ in length and $0.1 \mathrm{~mm}$ in width. They terminated on either side in a wider section, where a metallic interdigital transducer composed of 100 fingers was patterned. The period $p$ of the transducers was equal to 160,240 , or $320 \mathrm{~nm}$. These transducers are suitable for the generation 


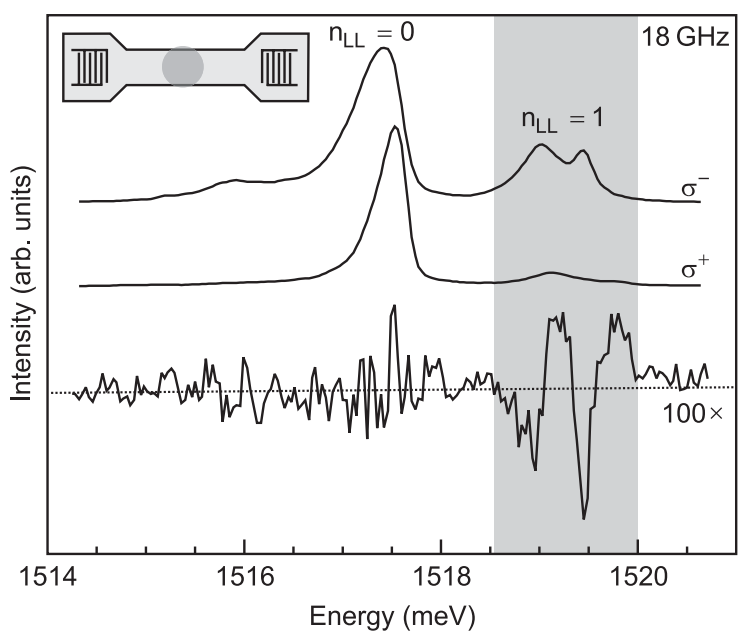

FIG. 1. Polarization resolved luminescence spectra (top trace, $\sigma^{-}$; middle trace, $\sigma^{+}$) for $n_{s}=8.8 \times 10^{10} \mathrm{~cm}^{-2}, B=1.3 \mathrm{~T}$ ( $\nu=2.8$ ), and a temperature $T=0.4 \mathrm{~K}$. The bottom curve shows the unpolarized differential luminescence spectrum in the presence of $18 \mathrm{GHz}$ SAWs. For the chosen density and $B$ field, resonant absorption takes place. The input power at one of the transducers is $1 \mathrm{~mW}$.

of SAWs with frequencies $f$ of either 18,12 , or $9 \mathrm{GHz}$. These frequencies correspond to wave numbers $k_{18}=$ $3.9 \times 10^{7} \mathrm{~m}^{-1}, \quad k_{12}=2.6 \times 10^{7} \mathrm{~m}^{-1}, \quad$ and $\quad k_{9}=1.95 \times$ $10^{7} \mathrm{~m}^{-1}$, respectively. Here, $k_{f}$ equals $2 \pi f / s$ with $s$ the velocity of sound in GaAs. Details of the SAW generation can be found in Ref. [10]. The SAW resonances typically have a width of $0.1-0.2 \mathrm{GHz}$. The SAW absorption at the electron spin resonance was detected by comparing the luminescence spectrum obtained in the absence of SAWs with the spectrum in the presence of SAWs. Subsequently, the measured spectra were subtracted to build a differential spectrum. The spectra were acquired with a CCD-camera and a double-grating spectrometer with a resolution of $0.03 \mathrm{meV}$. A stabilized semiconductor laser provided the continuous wave excitation. It was operated at $750 \mathrm{~nm}$ and delivered $0.1 \mathrm{~mW}$ of power across the active device area. The integral of the absolute value of the differential spectrum over the recorded spectral range is taken as a measure for the absorption amplitude of the SAWs.

Figure 1 depicts such a differential luminescence spectrum (bottom trace) under resonant conditions when an $18 \mathrm{GHz}$ wave travels along the mesa. The density $n_{s}$ is set to $8.8 \times 10^{10} \mathrm{~cm}^{-2}$ and a field of $1.3 \mathrm{~T}(\nu=2.8)$ is applied. The differential spectrum reveals a SAW induced redistribution of the electron population between 1.519 and $1.520 \mathrm{eV}$. To interpret this spectrum it is instructive to analyze circular polarization resolved luminescence data as the $\sigma^{+}$and $\sigma^{-}$polarization senses highlight the population of spin-down and spin-up electron levels, respectively. The luminescence curves for $\sigma^{+}$and $\sigma^{-}$circular polarization in the absence of SAWs are included in Fig. 1. Features associated with the lowest two electron Landau levels are easily identified. They appear in both spectra and are separated by an energy of $2.25 \mathrm{meV}$, the cyclotron energy at $1.3 \mathrm{~T}$. The signal involving the lowest electron Landau level $\left(n_{\mathrm{LL}}=0\right)$ has a similar intensity for both polarization directions. It reflects the zero net spin polarization of this completely filled Landau level. Conversely, the second partially filled Landau level appears only weakly in the $\sigma^{+}$polarization. It manifests the close to complete spin up polarization of the electrons accommodated in the second Landau level for a filling close to 3 . The spectral shifts of the levels and the fine splittings observed for different circular polarizations can be attributed to the energy spectrum of the photoexcited holes in the quantum well [12]. Returning to the differential spectrum, the shape and position of the feature between 1.519 and $1.520 \mathrm{meV}$ is now readily understood as a redistribution of the electrons among the two spin branches of the second Landau level, i.e., the electron spin resonance, and evidently no clear feature occurs for the completely filled lowest Landau level.

To corroborate further that such features in differential spectra originate from the ESR, the low electron density regime was investigated in detail. In this regime, the Coulomb interaction should only play a minor role compared to the Zeeman energy and the spin splitting is anticipated to coincide with the bare Zeeman energy as determined from the $g^{*}$ factor of GaAs, despite the transfer of nonzero momentum. Figure 2 plots the absorption amplitude (obtained by integrating the absolute value of the differential spectrum) as a function of $B$ for SAWs with frequencies of 18,12 , and $9 \mathrm{GHz}$. The density $n_{s}$ was chosen equal to $3 \times 10^{9} \mathrm{~cm}^{-2}$. The $B$ field at which each of the resonances occurs scales linearly with the SAW frequency. The proportionality constant between frequency and $B$ field is approximately equal to $0.45 \mu_{B} / h$, and hence the measured absolute value of $g^{*}$ is indeed close to the well-known value for GaAs (0.44). In order to account for

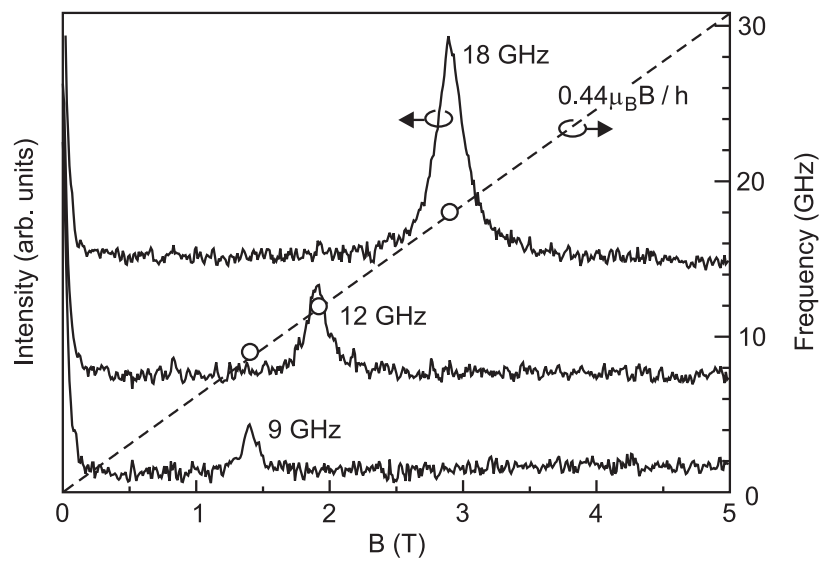

FIG. 2. Magnetic field dependence of the SAW absorption for 18,12 , and $9 \mathrm{GHz}$ at the low density of $3 \times 10^{9} \mathrm{~cm}^{-2}$. The input power at the transducer is approximately $1 \mathrm{~mW}$ for all frequencies. The extracted resonance positions agree well with those expected from the bare Zeeman energy (dashed line). 
the apparent excitation of spin-flip modes via the electric field associated with SAWs, it is necessary to invoke the spin orbit interaction [13]. For electrons in GaAs this coupling between the electric field controlled orbital motion and the spin degree of freedom is weak. The intensity of the differential spectra as in Fig. 1 is indeed very small and it should come as no surprise that the ESR absorption signal is about three to four orders smaller in magnitude in comparison with resonant absorption features related to plasmons and the cyclotron resonance detected previously with the same optical scheme under similar conditions [11].

Figure 3 illustrates the complex behavior of the SAW driven ESR as $n_{s}$ is increased and Coulomb interaction gains in importance. The SAW frequency is kept fixed at $18 \mathrm{GHz}$. The resonance located at $2.9 \mathrm{~T}$ for $n_{s}=$ $3 \times 10^{9} \mathrm{~cm}^{-2}$ shifts to much smaller $B$ fields and eventually for some densities even splits in two. Evidently, the spin-flip excitation energy at the SAW wave number $k_{18}$ is no longer solely determined by the Zeeman energy. The appearance of multiple resonances also reflects the nonmonotonic dependence with filling factor of the exchange contribution to the spin-flip excitation energy at nonzero wave number $[7,8]$. By plotting the position of the resonances in a $\left(n_{s}, B\right)$ plane with lines marking constant $\nu$ as in Fig. 4, the impact of exchange becomes particularly apparent. Because the entire data set is taken at a constant SAW frequency $f=18 \mathrm{GHz}$, the symbols describe contours of a constant spin-flip excitation energy of $h f$. At zero wave number or in the absence of exchange interaction these contours would reduce to a single horizontal line at a field equal to $2.92 \mathrm{~T}\left(h f / 0.44 \mu_{B}\right)$ (dashed line in Fig. 4). Instead, the contour lines are strongly repelled from the $\nu=1$ and $\nu=3$ lines. The SAW energy is insufficient to generate spin excitons at wave number $k_{18}$ near these fillings. Only at very low $n_{s}$ where the Coulomb interaction becomes negligible compared to the Zeeman energy or near filling $\nu=2$ where the system becomes unpolarized, the horizontal reference is approached. No SAW driven electron spin resonances are visible for $0.8<$ $\nu<1.2$ and $\nu>2$.9. Near filling 2, the optical method

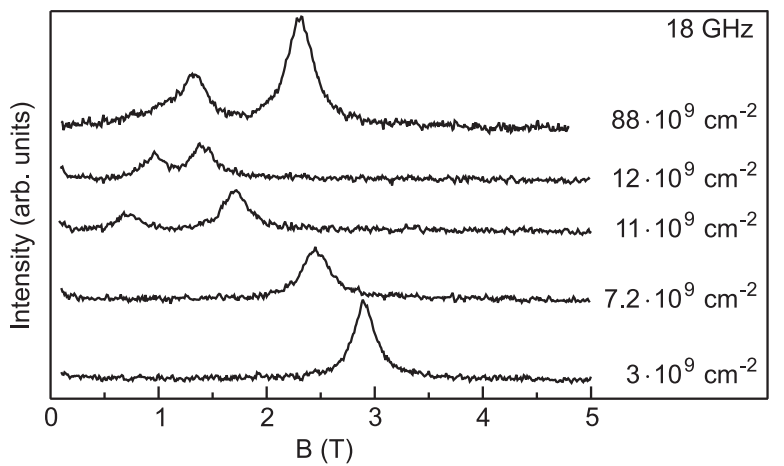

FIG. 3. Dependence of the SAW-absorption spectrum on electron density measured for a fixed SAW frequency of $18 \mathrm{GHz}$. looses its sensitivity as the luminescence signal stems nearly entirely from the unpolarized lowest Landau level. An alternative representation of the data is depicted in Fig. 5. It plots $\left|g^{*}\right|$ as a function of filling (Note that both $B$ and $n_{s}$ vary and hence also $k_{f} \cdot \lambda_{B}$ !). This graph includes also $12 \mathrm{GHz}$ data $\left(k_{12}=2.6 \times 10^{7} \mathrm{~m}^{-1}\right)$ for which many filling factors were also investigated. Clearly, the enhancement of the spin-splitting manifests itself stronger at larger wave numbers.

These experimental results are compared with a meanfield model of spin waves. It is an extension of the spinexciton theory developed earlier in Refs. [7,8], which focused on fillings close to integer values. The spin wave excitation energy $h \Omega$ is the sum of the Zeeman energy $E_{\mathrm{Z}}$ and the energy associated with the exchange interaction. In the mean-field approximation (i.e., neglecting possible correlation effects assuming that temperature is high enough), the latter contribution is proportional to the degree of electronic spin polarization, $x$, so that $h \Omega$ reads

$$
\begin{aligned}
h \Omega & =E_{z}+\frac{x}{2 \pi} \int_{0}^{\infty} V(q)\left[1-J_{0}\left(k q \lambda_{B}^{2}\right)\right]\left|F_{n}\left(q \lambda_{B}\right)\right|^{2} q d q \\
& \approx E_{z}+\frac{x}{8 \pi} k^{2} \lambda_{B}^{4} \int_{0}^{\infty} V(q)\left|F_{n}\left(q \lambda_{B}\right)\right|^{2} q^{3} d q,
\end{aligned}
$$

for $k \lambda_{B} \ll 1$. Here, $x=\nu_{n}$ for $0<\nu_{n}<1$ and $x=2-\nu_{n}$ for $1<\nu_{n}<2$, where $\nu_{n}$ denotes the filling of the highest occupied Landau level, $\left|F_{n}(z)\right|^{2}=e^{-z^{2} / 2}\left[L_{n}\left(z^{2} / 2\right)\right]^{2}$ is a Landau level specific factor [where $L_{n}(z)$ is a Laguerre polynomial] and $V(q)$ is the Fourier transform of the electron-electron interaction potential. For the bare Coulomb interaction, $V(q)=\frac{e^{2}}{\chi q}$, we obtain: $h \Omega=E_{z}+$ $\left(x e^{2} \lambda_{B} / 8 \pi \chi\right) k^{2} \beta_{n}$ with $\beta_{0}=\sqrt{\pi / 2}$ and $\beta_{1}=7 \beta_{0} / 4$. To compare the observed resonances in Fig. 5 with theoretical estimates, we evaluate the ratio $g^{*}=\frac{h \Omega}{\mu_{B} B}$ whenever the resonance condition $h \Omega=h f$ with $k=k_{f}$ holds. In the case of the bare Coulomb potential, we get

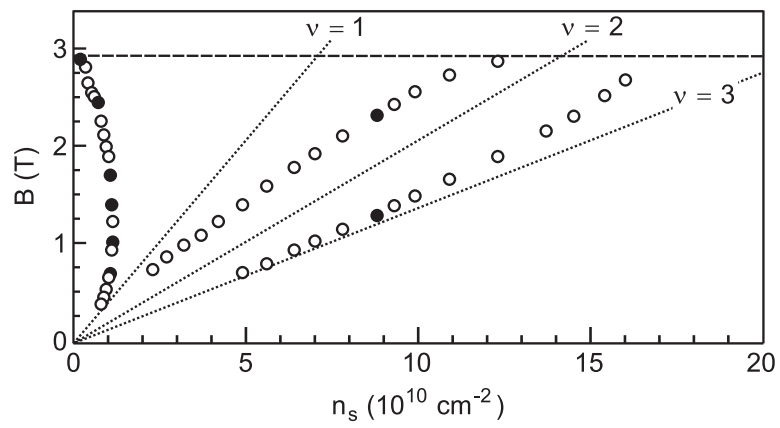

FIG. 4. Position of the electron spin resonances in the $B$ field vs $n_{s}$ plane for a SAW frequency of $18 \mathrm{GHz}$. The dashed line is the expected resonance position in the absence of exchange. Solid symbols are data points extracted from the absorption curves in Fig. 3. 


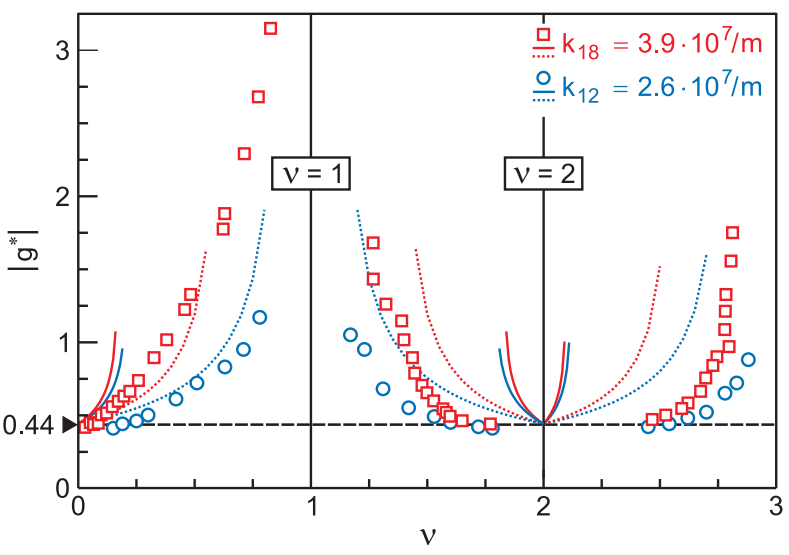

FIG. 5 (color). Effective " $g^{*}$ factor" of the spin wave, $g^{*}=$ $h \Omega / \mu_{B} B$ determined experimentally for $18 \mathrm{GHz}$ and $12 \mathrm{GHz}$ surface acoustic waves (square and circles) and theoretically using the bare Coulomb interaction (solid lines) and the 2D screened interaction (dotted lines). The blue color refers to the $12 \mathrm{GHz}$ results, and red to the $18 \mathrm{GHz}$ data.

$$
g^{*}=g_{0}+\beta_{n} \alpha g^{*}, \quad \alpha=\frac{x e^{2}}{4 \chi h s^{2}} \sqrt{\frac{h \Omega}{2 m_{e}}} .
$$

Solving this equation numerically for $g^{*}$ renders the solid curves in Fig. 5. The use of the bare Coulomb interaction in Eq. (1) clearly overestimates the exchange contribution to the spin wave energy. The resonance condition between the SAW and the spin waves can be met only for very small degrees of electronic spin polarization and the agreement with experiment is poor. In earlier thermal activation studies, it was also noticed that the activation energy measured in odd-integer quantum Hall states is less than the exchange energy evaluated using the bare Coulomb interaction $[4,5,14]$.

In recent studies of the exchange-enhanced $g^{*}$ factor and cyclotron gap in high-density $\mathrm{GaN} / \mathrm{AlGaN}$ heterostructures with large plasma parameter $r_{s}>1$ [15], it was pointed out that the measured activation energy gaps agree with values obtained under the assumption of 2D-screened Coulomb repulsion, $V_{\mathrm{scr}}(q)=\left(e^{2} / \chi\right) /\left(q+a^{-1}\right)$. In this case, the Coulomb interaction still goes as $1 / r$ at a distance less than the Bohr radius $a$, but is suppressed $\left(\sim a^{2} / r^{3}\right)$ at longer distances. Although for electrons filling only one Landau level this form of interaction overestimates screening, the doping layer displaced from the 2DES with the spacer and the potential existence of parallel conduction may also reduce the electron-electron interaction (it may curtail the Coulomb interaction at wave numbers equal to the inverse of the spacer width, which becomes particularly relevant at carrier densities below $10^{10} \mathrm{~cm}^{-2}$ where the average interparticle distance exceeds the spacer width). Below, we consider the 2D-screened interaction as the opposite extreme limit for estimating the exchange contribution to the spin wave dispersion. With the 2D screening length $a=\chi \hbar^{2} / m e^{2}$ corresponding to a fully polarized Fermi gas of electrons, we find the values of $g^{*}$ at the resonance condition between the SAWs and the spin waves for different fields and densities from a numerical solution of the following equation,

$$
g^{*}=g_{0}+\alpha g^{* 3 / 2} \int_{0}^{\infty} \frac{L_{n}\left(\frac{z^{2}}{2}\right) z^{3} e^{-z^{2} / 2} d z}{z+\sqrt{g^{*} \frac{\hbar}{a^{2} m_{e} \Omega}}} .
$$

The results are plotted as dotted lines in Fig. 5. They appear comparable, at low filling at least, to the experimental values of $\left|g^{*}\right|$ and lend support for significant screening of the electron-electron interaction. Even so, such an agreement may be fortuitous. As pointed out previously, the 2D screened Coulomb interaction should not apply at low fillings. Furthermore, the calculations do not describe properly the behavior at higher filling factors (between 1 and 3). There, the excitation energies in experiment are systematically lower than those for $\nu<1$ and substantially smaller than the calculated values. We can only speculate at present, but note that electron correlations in a partially filled Landau level are not included in the above mean-field analysis nor is the influence of density inhomogeneities.

In summary, we have successfully demonstrated the observation of ESR at nonzero wave numbers. A discrepancy with theory in the required energy has been disclosed. It can be mended only partially by invoking strong screening of the Coulomb interaction, whose origin, however, remains unresolved.

We acknowledge financial support from Max-Planck and Humboldt Research Grants, the Russian Fund of Fundamental Research, INTAS, the BMBF, and the DFG.

[1] D. Stein, K. von Klitzing, and G. Weimann, Phys. Rev. Lett. 51, 130 (1983).

[2] M. Dobers, K. von Klitzing, and G. Weimann, Phys. Rev. B 38, 5453 (1988).

[3] T. Ando, A. Fowler, and F. Stern, Rev. Mod. Phys. 54, 437 (1982).

[4] A. Usher, R. J. Nicholas, J. J. Harris, and C. T. Foxon, Phys. Rev. B 41, 1129 (1990).

[5] R. J. Nicholas, R. J. Haug, K. von Klitzing, and G. Weimann, Phys. Rev. B 37, 1294 (1988).

[6] I. V. Lerner and Yu. E. Lozovik, Zh. Eksp. Teor. Fiz. 78, 1167 (1980) [Sov. Phys. JETP 51, 588 (1980)].

[7] Yu. A. Bychkov, S. V. Iordanskii, and G. M. Eliashberg, Pis'ma Zh. Eksp. Teor. Fiz. 33, 152 (1981) [JETP Lett. 33, 143 (1981)].

[8] C. Kallin and B. I. Halperin, Phys. Rev. B 30, 5655 (1984).

[9] W. I. Dobrov, Phys. Rev. 134, A734 (1964).

[10] I. V. Kukushkin et al., Appl. Phys. Lett. 85, 4526 (2004).

[11] I. V. Kukushkin et al., Phys. Rev. Lett. 90, 156801 (2003).

[12] O. V. Volkov et al., Phys. Rev. B 56, 7541 (1997); JETP Lett. 65, 38 (1997).

[13] Yu. A. Bychkov and E. I. Rashba, J. Phys. C 17, 6039 (1984).

[14] V. T. Dolgopolov et al., Phys. Rev. Lett. 79, 729 (1997).

[15] W. Knap et al., J. Phys. Condens. Matter 16, 3421 (2004). 